تاثير يودر صمغ آنغوزه در مقايسه با آنتىبيوتيك بر عملكرد، ريختشناسى

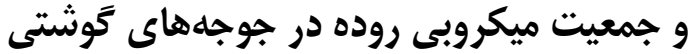

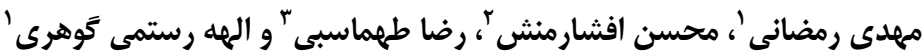

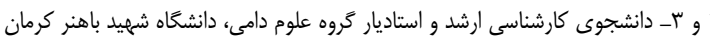

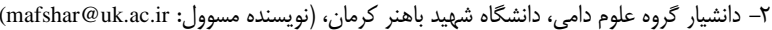 \\ تاريخ يذيرش: و

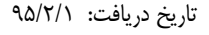

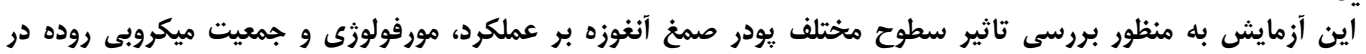

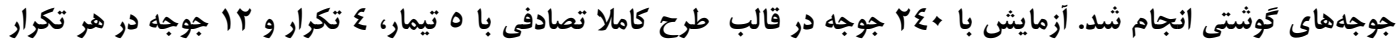

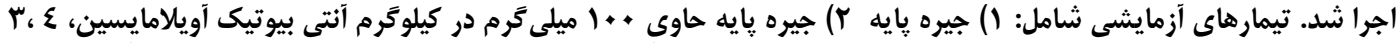

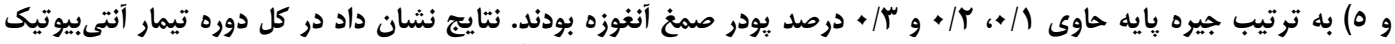

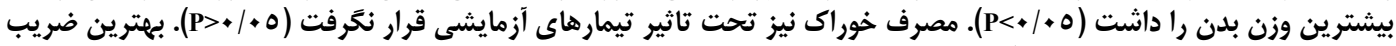

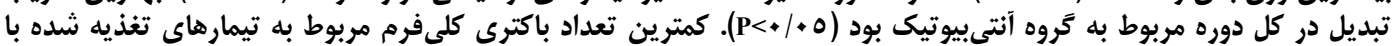

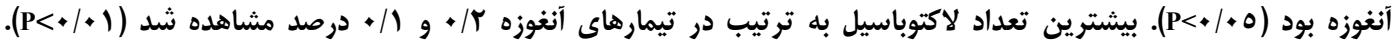

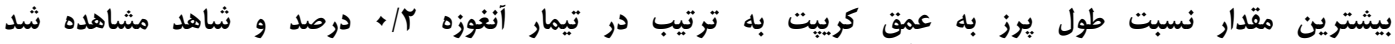

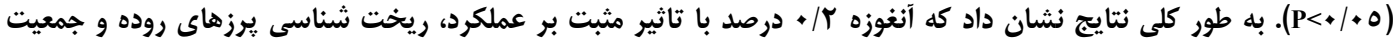

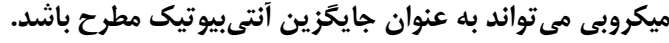

وازههاى كليدى: آويلامايسين، آنغوزه، جمعيت ميكروبى، مورفولوزى

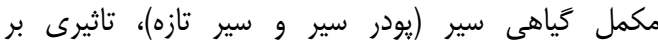

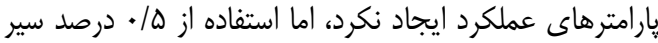

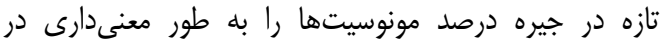

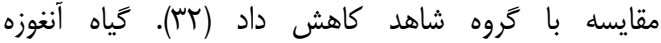
(Apiaceae) متعلق به خانوان (Ferula Asafoetida)

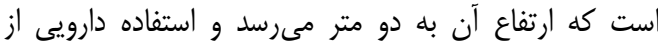

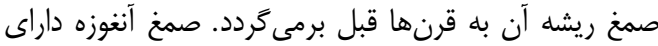

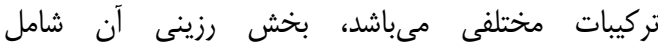

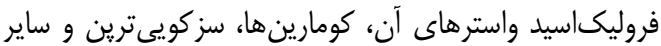

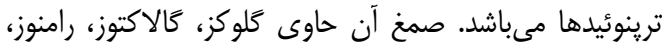

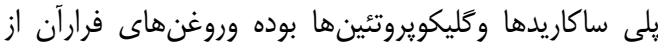

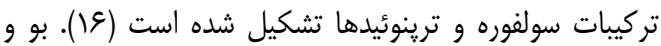

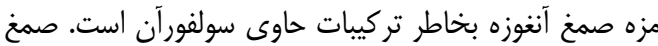

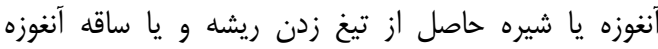

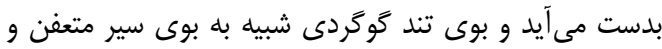

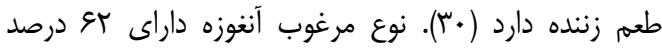

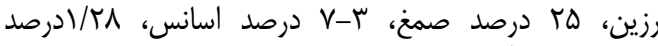

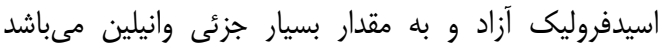

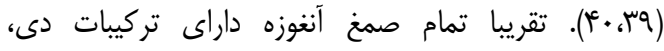

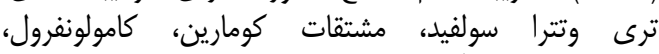

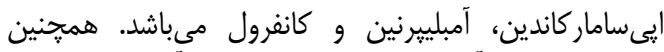

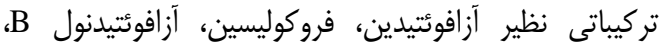

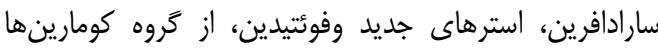

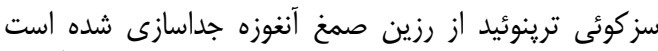

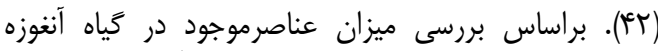
مشخص شده است كه عناصرى از قبيل آهن، استرانسيه،
استفاده از آنتىييوتيك ها يك امر رايج در دامبرورى بوده

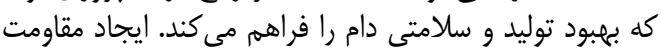

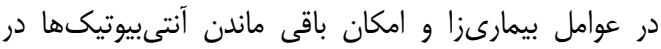

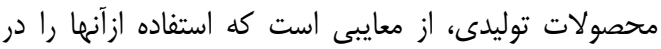

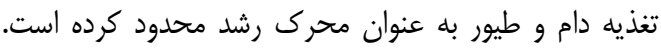

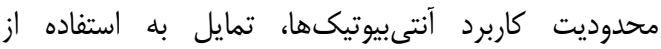

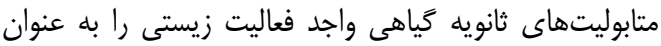

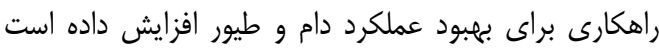

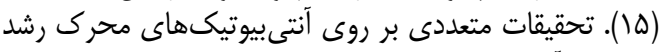

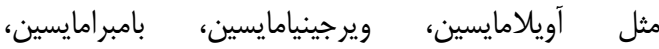

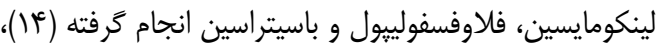

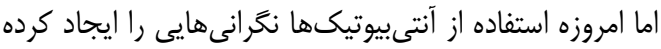

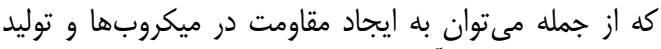

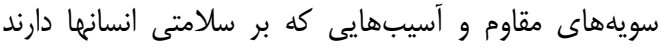

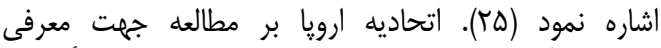

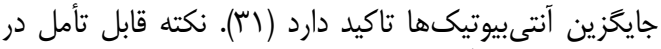

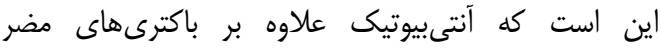

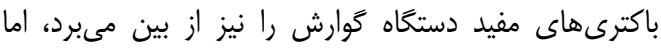

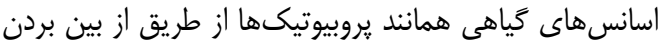

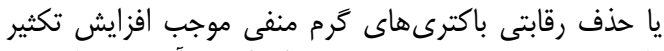

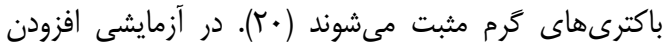

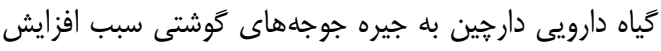

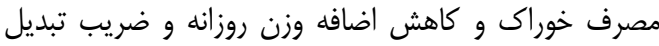

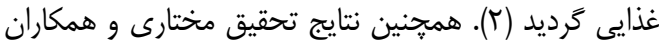
نشان داد كه تغذيه جوجههاى كوشتى با جيره حاوى (Y) 
به طور تصادفى انتخاب، كشتار و بلافاصله به آزمايشكاه

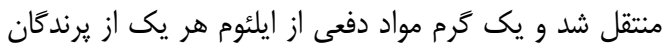

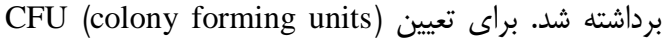
از روش شمارش قطرهاى، در محلول استريل بافر فسفات

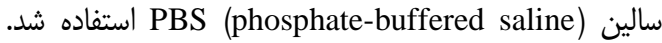

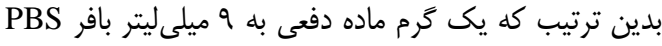

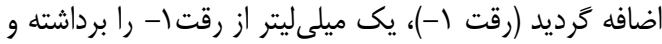

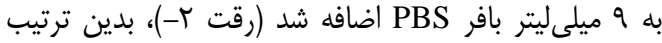

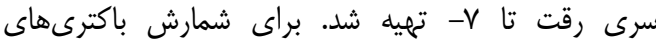
توليدكننده اسيد لاكتيك از محيط كشت

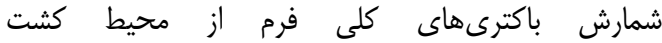
Mac Conkey

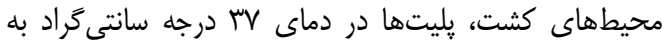

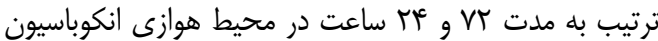

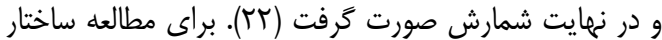

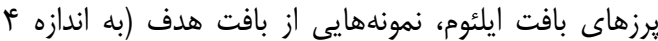

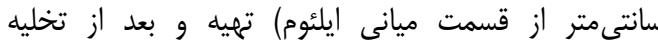

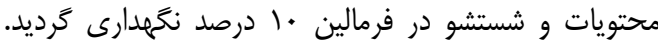

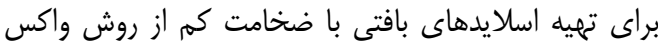

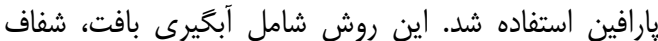

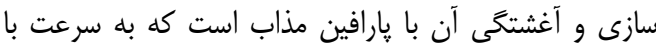

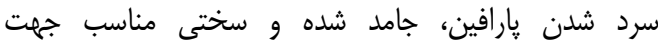

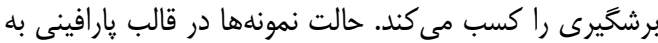

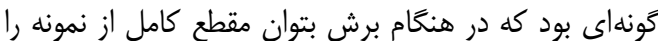

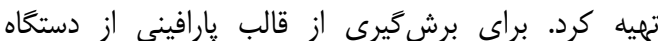

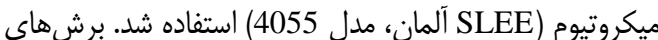

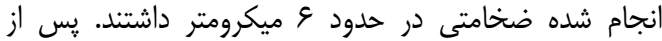

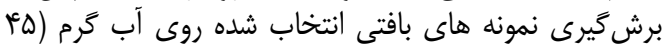

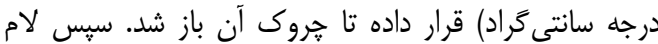

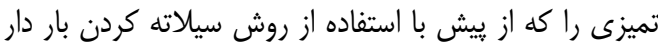

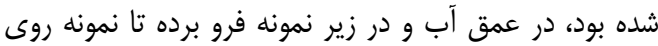

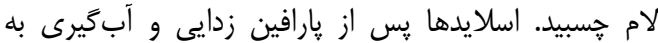

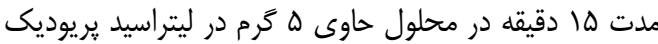

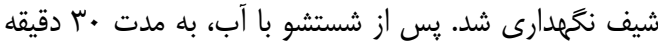

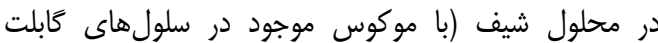

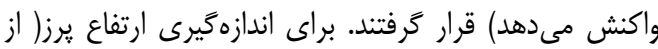

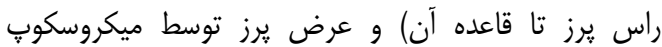

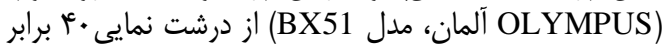

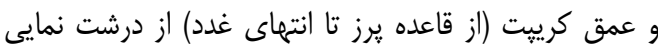

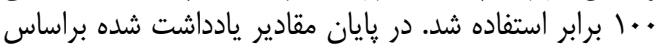

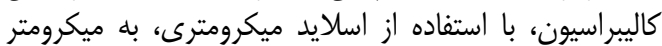

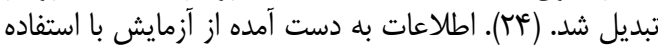

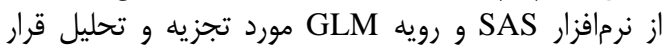

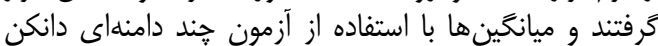
در سطح معنىدار ها درصد مورد مقايسه قرار كرَفيتند.
روى و مس به ميزان قابل توجهى دراين گياه موجود مى باشد

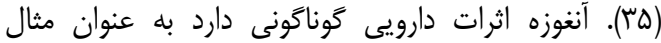

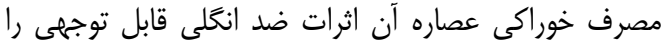

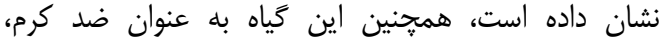

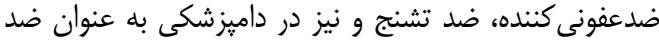

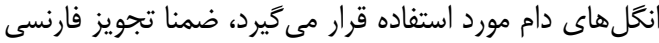

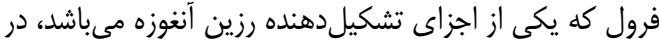

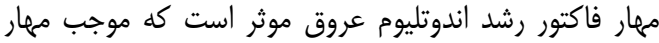

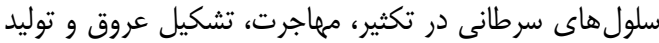

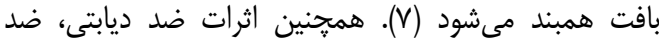

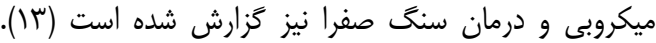

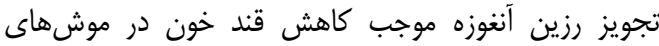

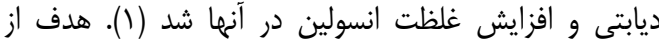

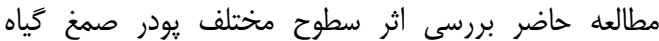

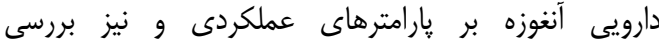

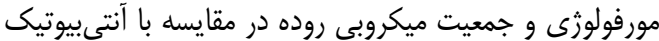

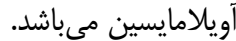

\section{مواد و روشها}

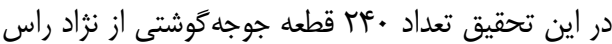

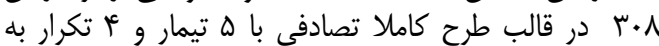

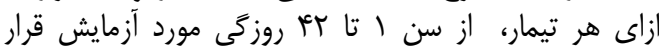

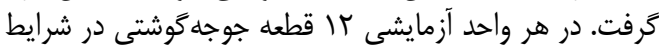

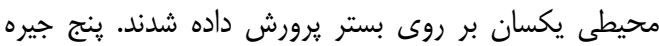

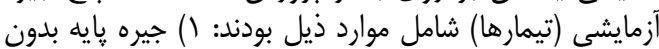

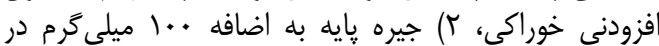

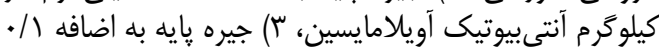

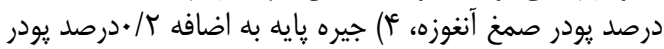

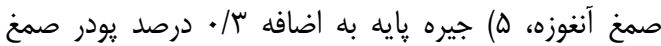

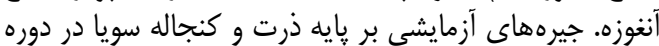

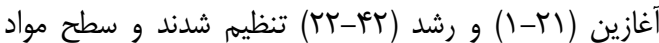

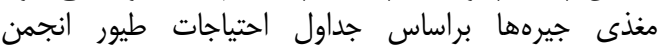

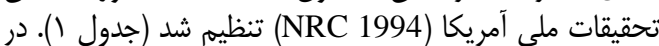

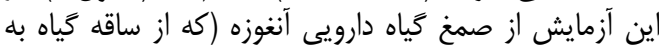

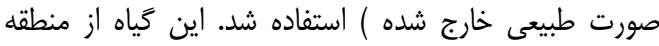

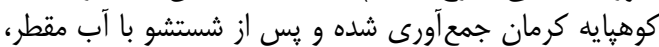

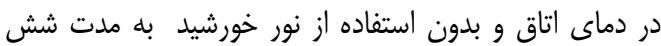

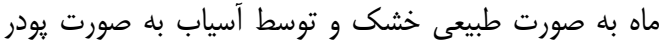

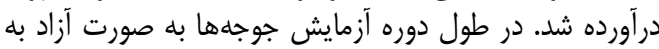

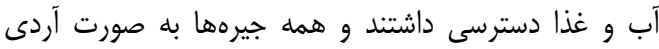

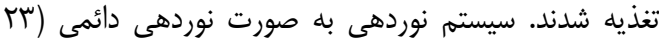

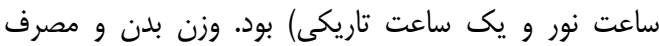

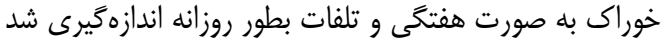

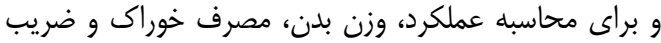

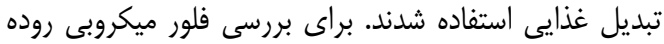
در سن آ روزگى يك يرنده از هر تكرار (أ برنده از هر تيمار) 

تاثير يودر صمغ آنغوزه در مقايسه با آنتىبيوتيك بر عملكرد، ريختشناسى و جمعيت ميكروبى روده در جوجههاى گوشتى

Table 1. Ingredients and composition of the basal diet in broiler chicks (\%)

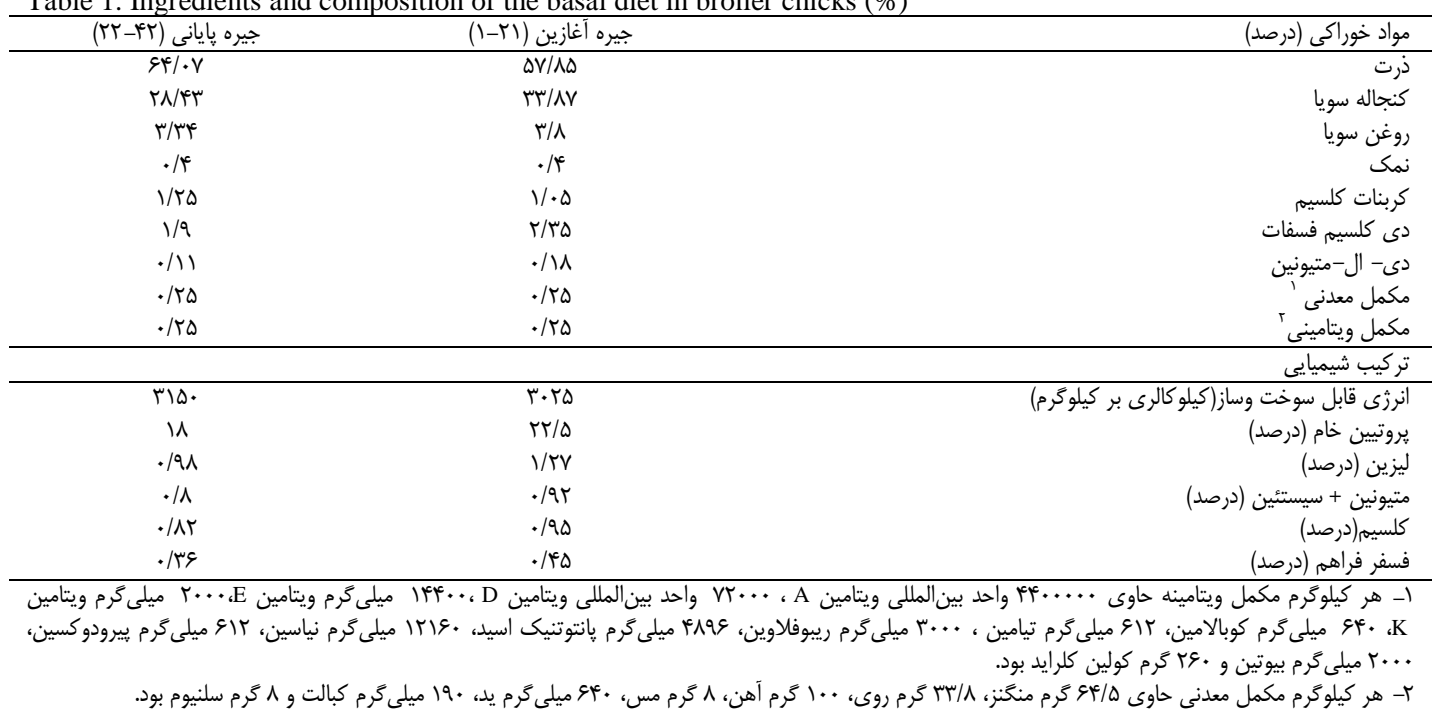

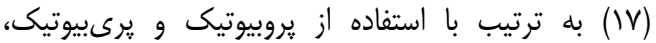

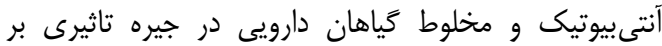

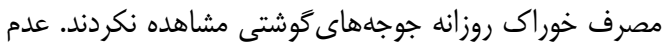

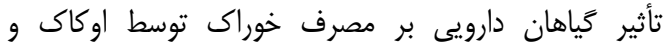

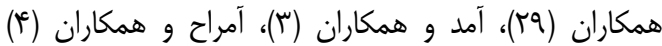

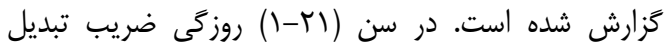

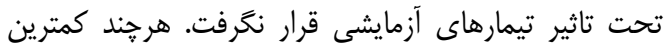

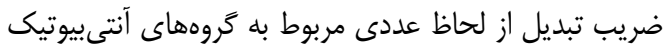

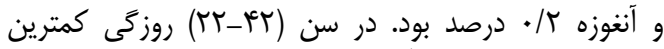

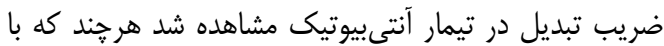

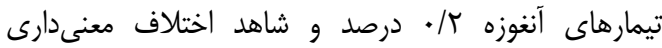

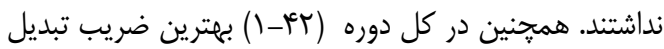

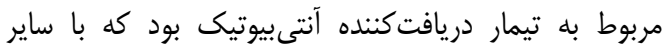

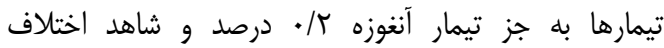

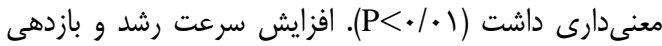

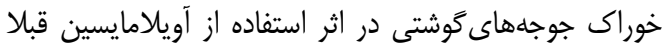

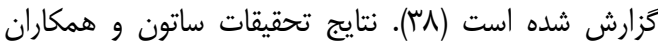

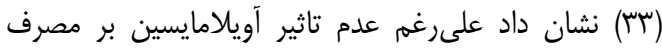

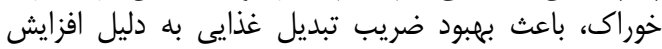

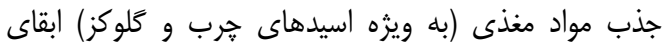

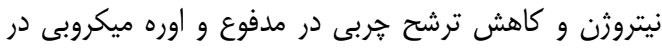

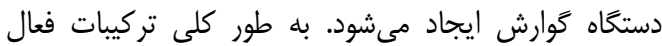

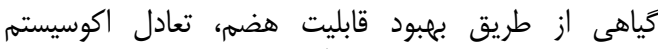

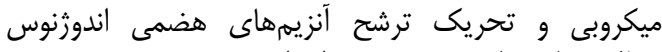

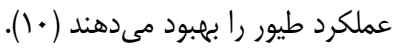

\section{نتايج و بحث عملكرد رشد}

نتايج مربوط به تأثير تيمارهاى آزمايشى بر وزن بلد بدن،

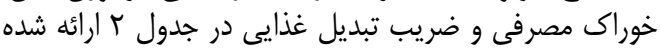

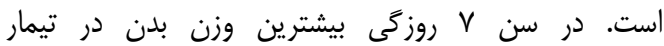

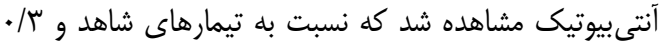

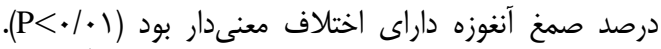

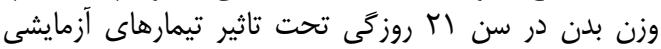

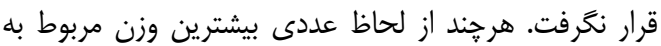

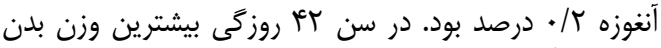

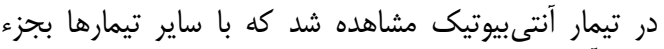

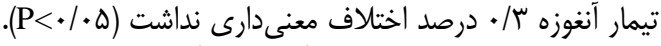

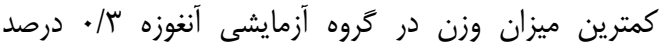

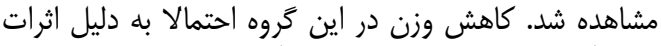

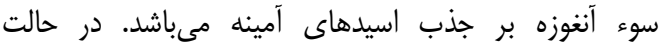

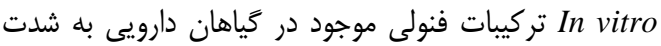

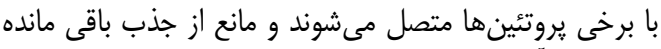

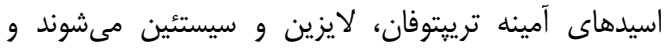

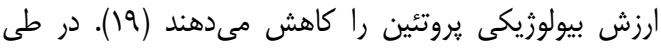

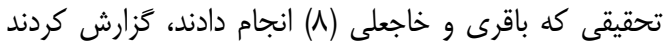

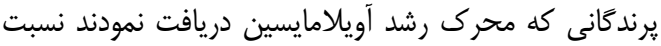

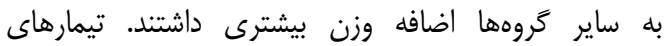

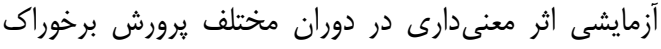

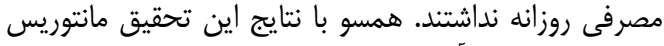

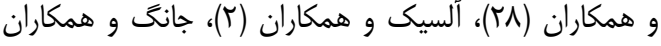


جدول r- تأثير تيمارهاى آزمايشى برعملكرد جوجههاى گَوشتى در دورههاى مختلف يرورش

Table 2. Effect of experimental treatments on broiler performance in different breeding periods

\begin{tabular}{|c|c|c|c|c|c|c|c|}
\hline \multicolumn{7}{|c|}{ تيمارهاى آزمايشى * } & \multirow{2}{*}{ دوروشهاى } \\
\hline P-Value & SEM & T5 & $\mathrm{T} 4$ & $\mathrm{~T} 3$ & $\mathrm{~T} 2$ & $\mathrm{~T} 1$ & \\
\hline & & & & & & \multicolumn{2}{|c|}{ وزن (گرم/ يرنده) } \\
\hline.$/+r$ & $r / ז q$ & $1 \% q / v q^{D}$ & $\mid f \Delta / r \cdot^{a}$ & $1 T V / r^{a b}$ & $\mid f N / \Gamma^{a}$ & $1 \% \cdot / .^{0}$ & 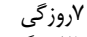 \\
\hline.$/ 11 f$ & ta/trt & VTF/TN & 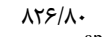 & $V \& V / r q$ & Var/ru & $V A T / \Delta F$ & ו آروزگى \\
\hline $.1 \cdot \mathrm{rV}$ & fl/ r & rrqr/A. & TFFI/DT & & arAD/ra & $r$ rVY/a. ${ }^{\text {abc }}$ & 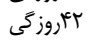 \\
\hline & & & & & & \multicolumn{2}{|c|}{ مصرف خوراك (كرم/ קرنده) } \\
\hline (III) & $1 / \cdot 9$ & $\mathrm{FV} / \mathrm{qg}$ & $\Delta I / \Lambda V$ & rq/Ft & $\mathrm{rq} / \mathrm{QV}$ & TN/TH & $|-r|$ \\
\hline ./9V8 & I/Ar & IQH/GT & IDT/TF & $\mid Q T / \Delta F$ & $\mid \Delta H / \Delta V$ & $10 T / 9 \varepsilon$ & th-tet \\
\hline . & $1 / \cdot 1$ & $a N / 4$ & १৭/४৫ & $91 / 98$ & QN/AT & $Q \Lambda / \Delta F$ & I Het \\
\hline & & & & & & \multicolumn{2}{|c|}{ ضريب تبديل (كرم/ گرم) } \\
\hline$\cdot 1 \cdot \sqrt{ } 9$ & שז./. & $1 / \uparrow \wedge$ & $1 / 49$ & שז/1 & $1 / 49$ & $1 / 41$ & $|-4|$ \\
\hline $.1+f^{c}$ & $.1 \cdot 11$ & $r / \cdot .^{a}$ & $r / \cdot \mu^{a b}$ & $r / \cdot .^{\mathrm{a}}$ & $1 / 99^{D}$ & $r / \cdot^{\mathrm{D}}$ & th-tet \\
\hline$\cdot 1 \cdots+1$ & $.1 \cdot 11$ & $1 / 19^{a}$ & $1 / \Lambda^{c}$ & $1 / \wedge \Delta^{D}$ & $1 / 19^{c}$ & $1 / 11^{\mathrm{Dc}}$ & 1 ter \\
\hline
\end{tabular}

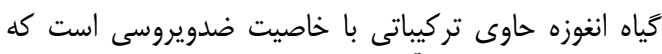
موجب مهار ويروس آنفولانزاى نوع A $\mathrm{A}$ مى شيود (N1H1).

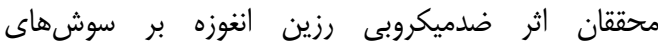

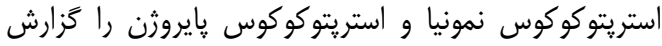

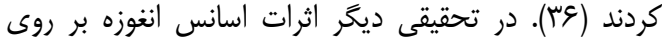

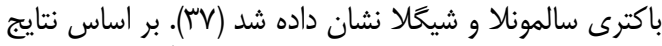

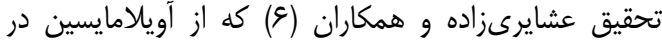

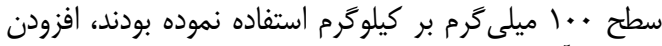

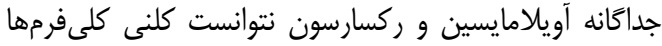
را به صورت معنى دارى كاهش دهان دهد، اما مخلوط آنها آنها كلنى اين

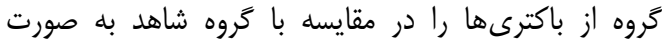

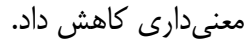

ريخت شناسى يرزهاى دادي روده

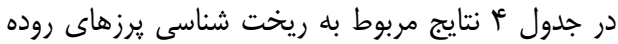

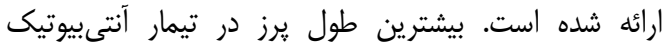

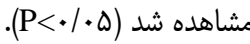

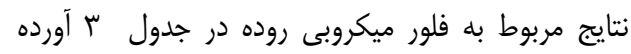

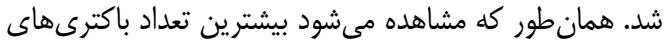

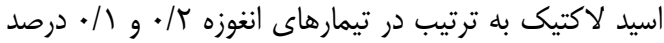

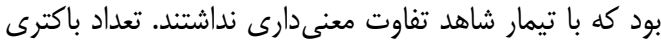

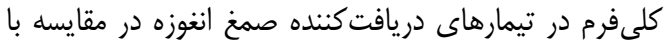

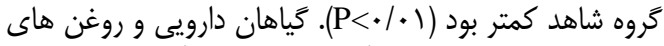

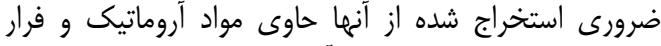

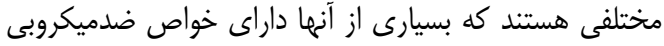

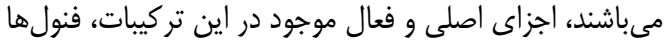

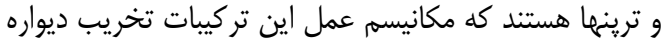

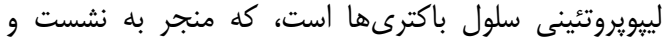

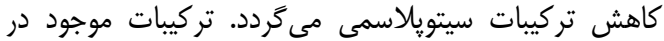

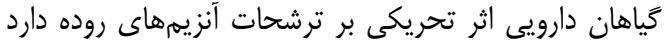

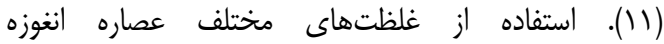

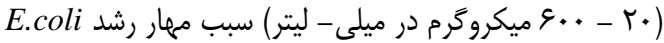

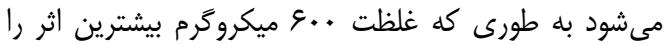

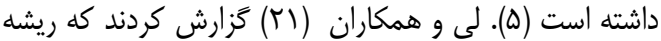

جدول بـ- تاثير تيمارهاى آزمايشى بر جمعيت ميكروبى روده جوجههاى گوشتى در سن ال روزگى Table 3. Effect of experimental treatments on intestine microbial population of broiler in 21 days of age

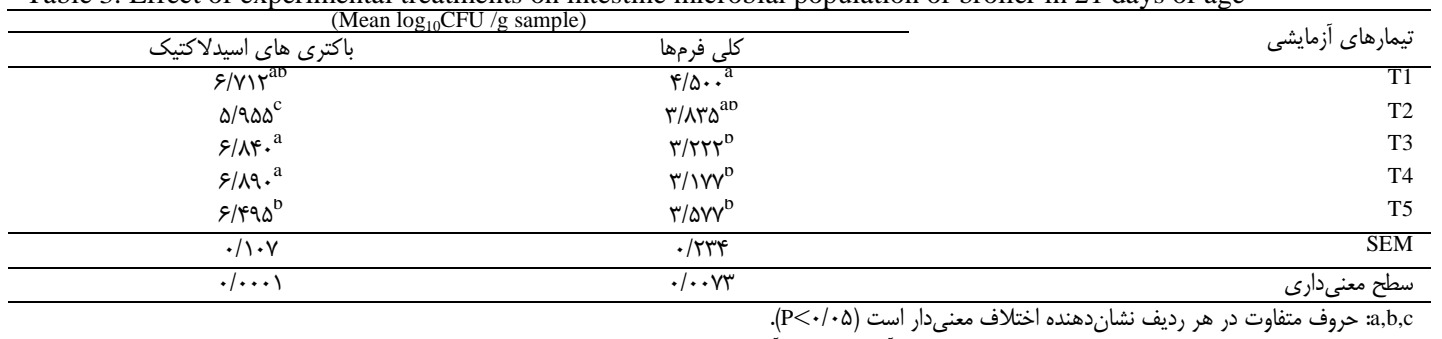

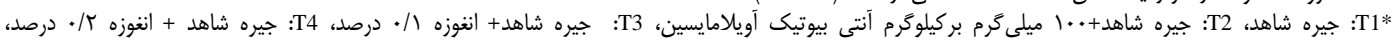

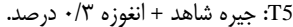



تاثير يودر صمغ آنغوزه در مقايسه با آنتى بيوتيك بر عملكرد، ريختشناسى و جمعيت ميكروبى روده در جوجههاى گوشتى

جدول זٔ- تاثير تيمارهاى آزمايشى مختلف بر مورفولوزى يرزهاى روده در جوجههاى گَوشتى Table 4. Effect of experimental treatments on the intestinal morphology in broiler chicks

\begin{tabular}{|c|c|c|c|c|}
\hline \multicolumn{4}{|c|}{ صفات مورفولوزى روده (ميكرومتر) } & \multirow[b]{2}{*}{ تيمارهاى آزمايشى } \\
\hline طول يرز /عمق كرييت & عمق كريبت & عرض برز & طول برز & \\
\hline $1 Q / 94 \cdot{ }^{a}$ & $1 \cdot N / \Delta \cdot^{0}$ & $1 Q F / Q \cdot .^{\circ}$ & IVTN/.." & $\mathrm{T} 1$ \\
\hline $\mid \leftarrow / \omega \cdot \Lambda^{D}$ & $\mid r a / \omega \cdot a$ & $198 / 0 . .^{\mathrm{a}}$ & $r \cdot r / / \Delta \cdot{ }^{a}$ & $\mathrm{~T} 2$ \\
\hline $1 \% / 994^{D}$ & $1 \cdot v / .^{0}$ & $1 \varepsilon r / \Delta \ldots^{a b}$ & $1 \% 98 / 00^{a}$ & $\mathrm{~T} 3$ \\
\hline $18 / 119^{a}$ & $111 / .^{\mathrm{D}}$ & $\mid \xi c / \Delta \ldots .^{a}$ & $\mid V Q \Delta / \Delta 0^{0}$ & $\mathrm{~T} 4$ \\
\hline $1 \% / \cdot v r^{c}$ & $1 \pi \Delta / \Delta \cdot \cdot^{a}$ & $\mid \Delta F / \Lambda \vee \Delta^{a b}$ & $I W \cdot / V Q^{D}$ & $\mathrm{~T} 5$ \\
\hline.$/ 491$ & $1 / 9.1$ & $r / \cdot r \cdot$ & $I T / I Q V$ & SEM \\
\hline$<\cdot / \cdots+$ &.$/ \cdots 1$ & $.1 \cdot 0$ &.$/ \cdots 1$ & سطح معنى دارى \\
\hline 0 & 1 & آويلاما & ا ميلى كرم اختا & 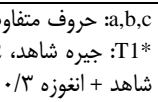 \\
\hline
\end{tabular}

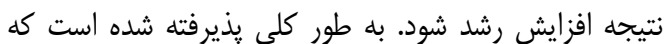

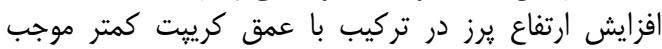

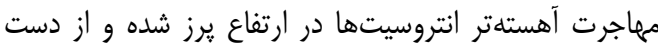

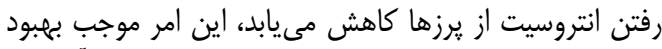

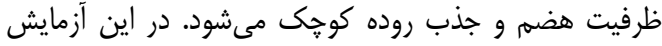

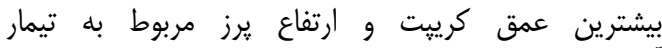

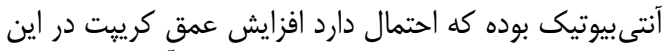

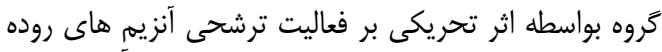

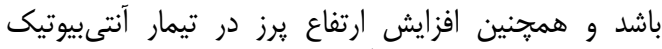

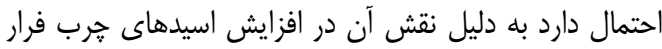

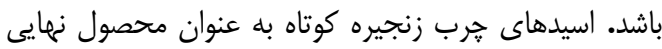

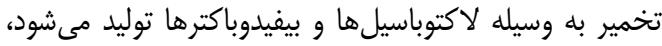

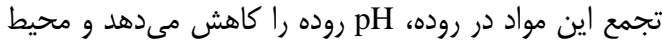

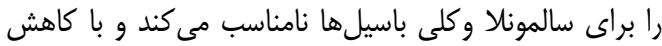

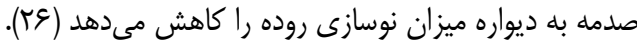

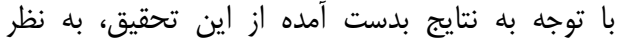

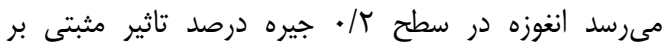

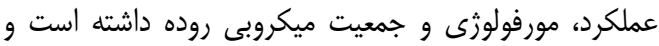

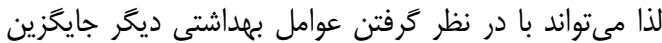
مناسبى براى آنتى بيوتيك باشد.
فلور ميكروبى روده همجنين طول برزها در تيمار هاى دريافت كننده / / • وس//.

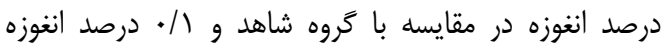

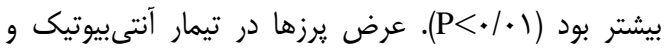

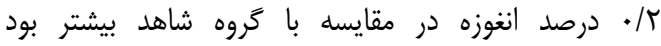

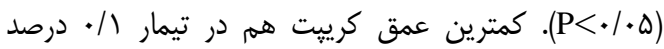

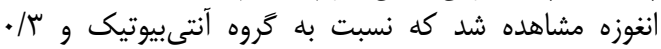

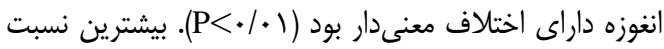

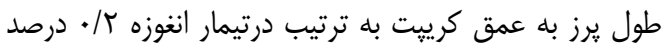

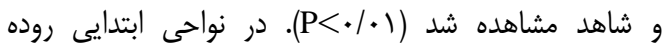

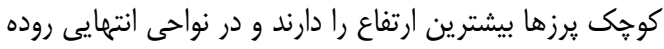

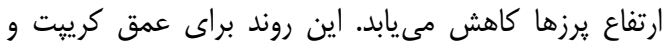

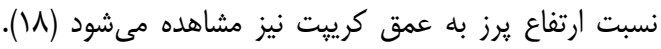

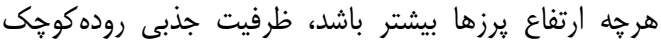

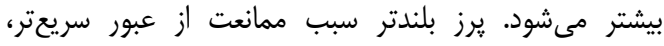

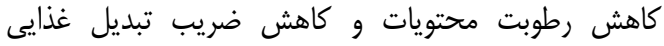

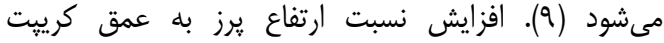

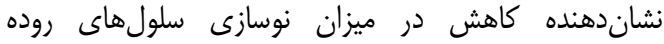

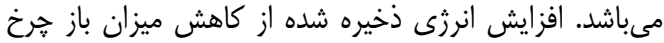

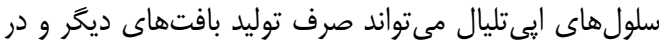


1. Abu-Zaiton A.S. 2010. Anti-diabetic activity of Ferula assa-foetida extract in normal alloxaninduced diabetic rats.Pakistan Journal of Biological Sciences, 4(2): 159-162.

2. Alcicek, A., M. Bozkurt and M. Cabuk. 2003. The effect of an essential oil combination derived from selected herbs growing wild in turkey on broiler performance. South African Journal of Animal Science, 33: 89-94.

3. Amad, A.A., K. Manner, K.R Wendler, K. Neumann and J. Zentek. 2011. Effects of a phytogenic feed additive on growth performance and ileal nutrient digestibility in broiler chickens. Journal of Poultry Science, 90: 2811-2816.

4. Amerah, A.M., G. Mathis and C.L. Hofacre. 2012. Effect of xylanase and a blend of essential oils on performance and Salmonella colonization of broiler chickens challenged with Salmonella Heidelberg. Journal of Poultry Science, 91: 943-947.

5. Amrita, V., D. Sonal and R. Shalin. 2009. Antibacterial Effect of herbs and Spices Extract on Escherichia coli. Electronic Journal of Biology, 5(2): 40-44.

6. Ashayerizade, O., B. Dastar, M. Shams Shargh and M. Khamiri. 2008. Study of intestinal microbial population and the response of young broiler diets supplemented with Roxarsone, Avilamycin and Formycin Gold.Journal of Science and Technology of Agriculture and Natural Resources, 43: 545-553 (In Persion).

7. Aydin, R., M. Karaman, T. Cicek and H. Yardibi. 2008. Black Cumin (Nigella sativa L.) supplementation into the diet of the laying hen positively influences egg yield parameters, shell quality, and decreases egg cholesterol. Poultry Science, 87: 2590-2595.

8. Bagheri, R. and Fkhajali. 2009. Avilamycin and probiotic effect on the compensatory growth of broiler chickens, following feeding with a diet low density. Journal of Science and Technology of Agriculture and Natural Resources, 48: 153-160 (In Persion).

9. Bradley, G.L., T.F. Savage and K.I. Timm. 1994. The effects of supplementing diets with Saccharomyces cervisiaevar. boulardi on male poult performance and ilealmorphology. Journal of Poultry Science, 73: 1766-1770.

10. Cross, D.E., R.M. McDevitt, K. Hillman and T. Acamovic. 2007. The effect of herbs and theiassociated essential oils on performance, dietary digestibility and gut microflora in chickens from 7 to 28 days of age. British Poultry Science, 48: 496-506.

11. Dorman, H.J.D. and S.G. Deans. 2000. Antimicrobial agents from plants: antibacterial activity of plant volatile oils. Journal of Applied Microbiology, 83: 308-316.

12. Eigner, D. and D. Scholz. 1999. Ferula asa-foetida and Curcuma longa in traditional medical treatment and diet in Nepal. Journal of Ethnopharmacology, 67: 1-6.

13. Fatehi, M., F. Fariftehand, Z. Fatehi-Hassanabad. 2004. Antispasmodic and hypotensive effects of Ferula asafoetida gum extract. Journal of Ethnopharmacology, 91(2-3): 321-324.

14. George, B.A., C.L. Quarles and D.J. Fagerberg. 1982 .Virginiamycin effects on controlling necrotic enteritis infection in chickens. Journal of Poultry Science, 61: 447-450.

15. Greathead, H. 2003. Plants and plant extracts for improving animal productivity. Proceeding of Nutrition Society journal, 62: 279-290.

16. http://WWW.ejbio.com/2009

17. Iranshahy, M. 2012. Traditional uses, photochemistry and pharmacology of asafetida (Ferula assafoetida oleo-gum-resin). Journal of Ethno pharmacology, 134: 1-10.

18. Jang, I.S., Y.H. Ko, S.Y. Kang and C.Y. Lee. 2007. Effect of commercial essential oil on growth performance, digestive enzyme activity and intestinal microflora population in broiler chickens. Journal of Animal Feed. Journal of Science Technology, 134: 305-315.

19. KarimiTarshizi, M.A. 2005. Isolation, identification and selection of lactic acid bacteria for the production of probiotics in broilers, Ph.D. dissertation, Faculty of Agriculture, Tarbiat Modares University (In Persion).

20. Kreydiyyeh, S.I., J. Usta and R. Copti. 2000. Effect of cinnamon, clove and some of their constituents on the NA+-K+- ATPase activity and alanine absorption in the rat jejunum. Journal of Food and Chemichal Toxicology, 38: 755-762.

21. Kumar, S., K.C. Sharadama and P.M. Radhakerishna. 2010. Effects of Garlic active basedgrowth promoter on growth performance and specific pathogenic intestinal microbial counts of broiler chicks. Indian Journal of Poultry Science, 9: 244-246.

22. Lee, C.L., L.H. Cheng, C.C. Liaw, M.H. Abd El-Razek, F.R. Chang and Y.C. Wu. 2009. Infoluenza a (N1H1) antiviral and cytotoxic agents from Ferula assa-foetida.Journal of natural products, 30(20): $1568-1572$.

23. Li, Y.L. 1991. Culture Medium Manual (Changchun, China, Jilin Science and Technology Press).

24. Lopez, A. 1998. AssaFoetidin and ferocolicin, two sesquiterpenoidcumarins from Ferula assafoetida.Journal of Tetraherdon letters, 29(13): 1557-1560.

25. Mc, J.F. 1948. Histological and histochemical uses of periodic acid. Journal of Stain Technology, 23: 99-108.

26. Mccartney, E. 2002. The natural empire strcks book. Journal of Poultry International, 41(1): 36-42.

27. Mohan, K.O.R and C.K. James. 1988. The role of Lactobacillus sporogens (probiotic) as feed additives. Journal of Poultry Guide, 25: 37-39.

28. Mokhtari, A., M.R. Akbari and E. Asadi Khoshoei. 2016. Effect of garlic powder or fresh ground garlic on performance and immune response of broiler chickens. Research on Animal Production, 13: 24-31 (In Persian). 
rr تاثير يودر صمغ آنغوزه در مقايسه با آنتىبيوتيك بر عملكرد، ريختشناسى و جمعيت ميكروبى روده در جوجههاى گوشتى .

29. Mountzouris, K., H. Beneas, P. Tsirtsikos, E. Kalamara and K. Fegeros. 2006. Evaluation of the effect of a new probiotic product on broiler performance and cecalmicroflora composition and metabolic activities. International Poultry Scientific Forum Atlanta, Georgia, January, 16: 23-24.

30. Ocak, N., G. Erener, F. Burak, M. Sungu, A. Altop and A. Ozmen. 2008. Performance of broilers fed diets 6 supplemented with dry peppermint (Menthapiperita L.) or thyme (Thymus vulgaris L.) leaves as growth 7 promoter source. Journal of Animal Sciences, 53: 169-175.

31. Raghavan, S. 2007. Handbook of spices: seasonings and flavorings. (3nd ${ }^{\mathrm{ed}}$.).CRC press. USA, pp: 6970.

32. Rahmatnejad, E., H. Roshanfar, O. Ashayerizadeh, M. Mamooee and A. Ashayerizadeh. 2009 Evolation the effect of several non- antibiotic additives on growth performance of broiler chickens.Journal of Animal and Veterinary Advances, 8: 1670-1673 (In Persian).

33. Shirzadegan, K. 2016. The impact of different levels of cinnamon powder (cinnamomum veru) on performance, blood metabolites and inner organs weight of broilers. Research on Animal Production, 13: 16-23 (In Persian).

34. Sutton, A.L., J.C. Nye, J.A. Patterson, D.T. Keay and E.J. Furumoto-Elkin. 1989. Effects of avilamycin in swine and poultry wastes on methane production in anaerobic digesters. Journal of Biological Wastes, 30: 35-45.

35. Tarlow, D.M., P.A. Watkins, R.E. Reed, R.S. Miller, E.E. Zwergel and M.D. Lane.1977. Lipogenesis and the synthesis and secretion of very low density lipoprotein by avian liver cells in nonproliferating monolayer culture. Journal Cell Biology, 173: 332- 353.

36. Tucker, A.O. and T. DeBaggio. 2009. The Encyclopedia of Herbs: A comprehensive reference to herbs of flavor and fragrance. Timber press. USA. pp: 236-237.

37. Unasho, A., A. Geyid, A. Melaku, A. Debela, A. Mekasha, S. Girma, T. Kebede, S. Fantaw, NAsaminew, K. Mamo and J. Med. 2009 .Investigation of antibacterial activities of Albiziagummifera and Ferula communis on Streptococcus pneumoniae and Streptoccuspyogenes Ethiopian medical journal, 47(1): 25-32.

38. Vaishnavi, C., S. Kaur and M. Kaur. 2007. Bactericidal activity of kitchen spices and condiments on enteropathogens. Journal of Natural product radiance, 6: 67-75.

39. Van Campenhout, J., J. Van Hemel, K. Vandenkerckhov, K. Mollen and B. Sas. 2001. Performance of an alternative to antibiotics in broiler with high intestinal count of clostridium peferingens, Proc. $13^{\text {th }}$ Euroupean Symptoms Poultry Nutrition, pp: 127-128.

40. Vijn, J.P. 1982. Carlyle and Jean Paul: their spiritual optics. John Benjamins Publishing Company. Germany, pp: 135-140.

41. Wyk, B.E. and M. Wink. 2004. Medicinal plants of the world: an illustrated scientific guide to important medicinal plants and their uses. Timber Press. USA, pp: 221-223.

42. Yesodharan, K. and K.A. Sujana. 2007. Wild edible plants traditionally used by the tribes in the Parambikulam Wildlife Sanctuary, Kerala, India. Journal of Natural product radiance, 6(1): 74-80.

43. Zare, AR., M. Omidi, H. Fallah Hoseini, D. Yazdani, S.H. Rezazadeh, N. Iravani and A. Oladzadeh. 2011. A review of the pharmacology of medicinal plants Ferula assa-foetida,Journal of Medicinal Plants, 40: 17-25 (In Persian). 


\title{
The Effect of Ferulaassa-Foetida Gum Powder Compare to Antibiotic on Performance, Microbial Population and Intestinal Morphology in Broiler Chickens
}

\author{
Mahdi Ramezami ${ }^{1}$, Mohsen Afsharmanesh ${ }^{2}$, Reza Tahmasbi ${ }^{3}$ and \\ Elahe Rostami Gohari ${ }^{1}$ \\ 1 and 3- M.Sc. Student and Assistant Professor, Department of Animal Science, Shahid Bahonar University of \\ Kerman \\ 2- Associate Professor, Department of Animal Science, Shahid Bahonar University of Kerman, \\ (Corresponding author: mafshar@uk.ac.ir) \\ Received: 20 April $2016 \quad$ Accepted: 26 December 2016
}

\begin{abstract}
This experiment was conducted to investigate the effect of different levels of Ferulaassafoetida gum powder and antibiotic on performance, intestine morphology and microbial population in broiler chicks. The experiment was carried out in a completely randomized design with 240 chicks in 5 treatments, 4 replicates and 12 chicks per replicate. Treatments included: 1) basal diet without additives, 2) basal diet containing $100 \mathrm{mg}$ per $\mathrm{kg}$ of antibiotics Avilamycin.3, 4 and 5) basal diet contain $0.1,0.2$ and 0.3 Ferula assa-foetida gum powder, respectively. The results showed that in whole experiment period, treatment fed antibiotic had the highest body weight $(\mathrm{P}<0.05)$. Feed intake was not affected by treatments. The best feed conversion in whole period was related to antibiotic group. The lowest number of coliform bacteria was related to treatments were fed with Ferula assa-foetida. The highest number of Lactobacillus were seen, in 0.2 and 0.1 percent Ferula assa-foetida treatments respectively that had significantly different with other treatments except control $(\mathrm{P}<0.01)$. The highest ratio of villus length to crypt depth has found for 0.2 percent of Ferula assa-foetida and control group.
\end{abstract}

Keywords: Avilamycin, Ferula assa-foetida, Morphology, Microbial population 